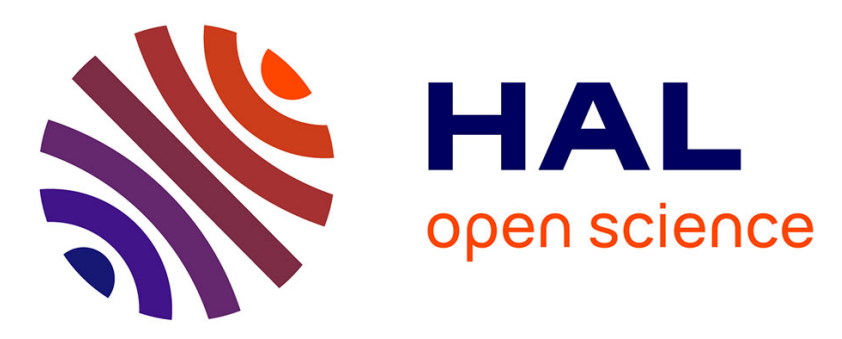

\title{
Smith-Predictor-Based Torque Control of a Rolling Diaphragm Hydrostatic Transmission
}

Marco Bolignari, Gianluca Rizzello, Luca Zaccarian, Marco Fontana

\section{To cite this version:}

Marco Bolignari, Gianluca Rizzello, Luca Zaccarian, Marco Fontana. Smith-Predictor-Based Torque Control of a Rolling Diaphragm Hydrostatic Transmission. IEEE Robotics and Automation Letters, 2021, 6 (2), pp.2970-2977. 10.1109/LRA.2021.3062310 . hal-03202791

\section{HAL Id: hal-03202791 \\ https://hal.laas.fr/hal-03202791}

Submitted on 20 Apr 2021

HAL is a multi-disciplinary open access archive for the deposit and dissemination of scientific research documents, whether they are published or not. The documents may come from teaching and research institutions in France or abroad, or from public or private research centers.
L'archive ouverte pluridisciplinaire HAL, est destinée au dépôt et à la diffusion de documents scientifiques de niveau recherche, publiés ou non, émanant des établissements d'enseignement et de recherche français ou étrangers, des laboratoires publics ou privés. 


\title{
Smith-predictor-based torque control of a rolling diaphragm hydrostatic transmission
}

\author{
Marco Bolignari ${ }^{1}$, Gianluca Rizzello ${ }^{2}$, Luca Zaccarian $^{3}$, Marco Fontana $^{4}$
}

\begin{abstract}
Rolling Diaphragm Hydrostatic Transmissions (RDHT) are high-performance low-cost solutions to delocalize heavy actuators away from the joints of robotic systems. Exploiting a low-cost pressure-based sensing technique, we propose here a Smith-predictorbased joint torque control of an RDHT-based actuation system. We also use a load-cell sensor for ground truth validation. The developed feedback controller is conveniently tuned based on an arbitrary pre-specified closed-loop natural frequency and damping ratio. This preserves the open-loop bandwidth while removing the intrinsic oscillations of the lightly damped highly transparent open-loop plant. Experimental tests validate the proposed control strategy, both in a stand-alone torque setpoint configuration and in a series of Human-Robot-Interaction tests confirming the significant advantages of the closed-loop control architecture.
\end{abstract}

\section{INTRODUCTION}

Developing lightweight robotic systems for increased compliance and mild requested power, typically associated with reduced moving masses/inertia [10], has been long recognized to be associated with successful remotization of the actuators. To this end, heavy actuators are placed at a zero-speed location (e.g., the robot base), far from the joint axes; a direct-drive motorization is then feasible, i.e. no reduction stage is used, which is known to minimize the inertia reflected to the robotic link, thus resulting in maximized bandwidth and safe interaction. The problem is then shifted to developing power transmission technologies for transferring the torque from the base actuators to the actuated joint. A range of solutions have been proposed in the past years (see, e.g., [6], [8], [9], [12]), but an effective recent technology named rolling diaphragm hydrostatic transmission (RDHT) [22] has several advantages in terms of friction and backlash. Different RDHT layouts have then been proposed for the actuation of fully-passive robotic systems [21], lower limb exoskeletons [18], robotic grippers [15] and MR compatible robots [4].

Among other strong potentials of RDHT, a rather unexplored path is their use for the actuation of high-bandwidth human-robot interaction (HRI), with intrinsic compliance features emerging from their stiffness/bandwidth characteristics. Indeed, existing HRI approaches mostly rely on standard robotic technologies combined with cautious impedance control laws conservatively guaranteeing stability for any contact condition (see, e.g., [1], [5], [7] and references therein). A first step towards this end is certainly to investigate the currently unexplored challenge of providing a high-bandwidth non-oscillatory torque response despite the underdamped oscillatory behavior typically generated by the low friction RDHT devices. This goal inevitably requires some form of feedback control loop to artificially inject damping in the actuator dynamics. Feedback control has been used to this end in [13] when using Series-Elastic Actuators (SEA), which also offer an intrinsic compliant behavior. The corresponding control solutions, however, when applied to our RDHT systems, whose dynamics is quite different due to the presence of fluid lines, revealed to be experimentally less effective than the Smith-predictor-based solution proposed in this paper.

An appealing feature of the RDHT architecture, already mentioned in [22], is that the torque feedback measurement can be conveniently implemented by a differential pressure measurement on the fluid lines, a cost-effective paradigm validated experimentally in [20]. The measurement positioning along the fluid lines poses an interesting trade-off between accuracy and stabilizability wherein a proximal placement, closer to the actuator (colocation), is more stable but less accurate, and a distal placement is more accurate but exhibits increased dynamical effects.

In this work, we reach beyond the preliminary results of [20] by exploiting the high-performance RDHT device that we recently developed in [3]. This device is an ideal testbed not only for validating the high-bandwidth high-transparency RDHT torque transmission paradigm, but also for investigating the maximum performance limits that one can reasonably expect from RDHTs. The considerably low friction characterizing our experimental device poses nontrivial control design challenges, because of the highly oscillatory open-loop response. The techniques proposed in this paper apply to general RDHT architectures with low friction, both in the floating-bonnet and the floating-piston layouts, since they rely on the same working principle.

Manuscript received: October, 22, 2020; Revised December, 19, 2020; Accepted February, 06, 2021.

This paper was recommended for publication by Editor Clement Gosselin upon evaluation of the Associate Editor and Reviewers' comments.)

This work was supported by MIUR under the Program Department of Excellence, of the DII, University of Trento and Scuola Superiore Sant'Anna

${ }^{1} \mathrm{M}$. Bolignari is with the Department of Industrial Engineering, University of Trento, Trento 38123, Italy marco.bolignari@unitn.it

${ }^{2}$ G. Rizzello is with the Dept. of Systems Engineering and the Dept. of Material Science and Engineering, Saarland University, Saarbrücken, DE 66123 Germany gianluca.rizzello@imsl.uni-saarland.de

${ }^{3}$ L. Zaccarian is with LAAS-CNRS, Université de Toulouse, CNRS, Toulouse, France, and Dip. di Ing. Industriale, University of Trento, Italy.

${ }^{4}$ M. Fontana is with the TeCIP Institute, Scuola Superiore Sant'Anna, Pisa, 56100, Italy marco.fontana@santannapisa.it

Digital Object Identifier (DOI): see top of this page. 

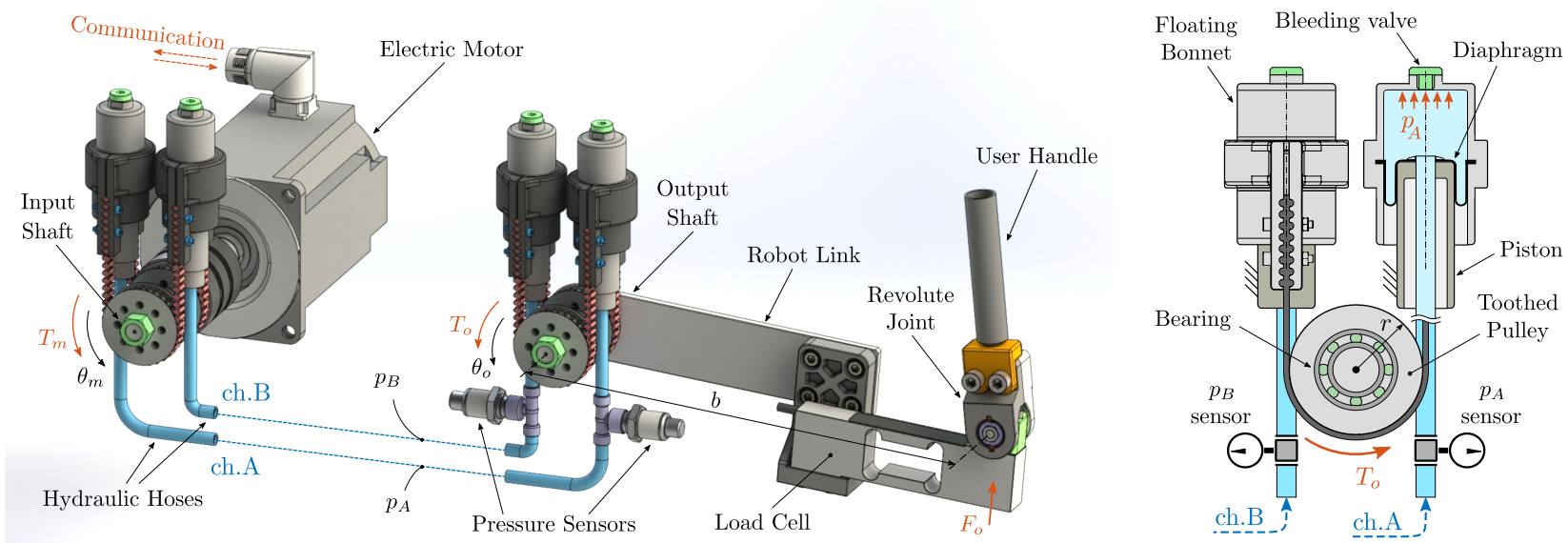

Fig. 1. Rolling diaphragm hydrostatic transmission. Left: setup allowing the human user to interact with the actuation system through the handle; alternatively, the handle can be removed and the load cell fixed to the ground. Right: scheme of the working principle of the output joint.

Within this setting we provide in this paper several contributions listed next, together with the corresponding paper organization: 1) We confirm the high accuracy of the torque feedback based on distal pressure measurement by using an extra ground truth load-cell sensor for validation purposes (as reported in Sect. II). 2) In Sect. III, through extensive experimentation with different hose lengths, we characterise the dynamic response of the open-loop transmission, along with a linearity analysis. Then we propose and identify, for all of the considered hose lengths, a parametric linear dynamical model for the openloop system, comprising a combination of a second-order underdamped response and a time delay, and achieving a desirable trade-off between complexity and accuracy (alternative existing models, for example, comprise a larger number of states [20]). 3) We propose and experimentally validate a parametric feedback control design strategy, reported in Section IV, based on a Smith-predictor [14] together with a dynamic controller automatically tuned to achieve arbitrary user selected closed-loop natural frequency and damping ratio; the closed loop then reaches almost the same natural frequency as the open-loop plant, in spite of the large amount of artificial damping introduced by the control scheme. 4) Finally, in Section V we provide an encouraging preliminary illustration of the advantages arising from the proposed controlled torque transmission device in human robot interaction by simple tasks performed by a human operator.

\section{ROLLING DiAPHRAGM Hydrostatic TRANSMISSION}

\section{A. Working Principle}

Fig. 1 (left) shows the main elements of the RDHT actuation system proposed in our previous work [3]. The electric motor on the left, connected to the input shaft, provides the actuation torque. Two hydraulic hoses couple the input and output shafts. Two pressure sensors are placed at the end of the hoses, close to the output shaft, where a robot link is connected. A load cell is located at the end of the link to measure the actual force $F_{o}$ exchanged between the actuation mechanism and an external element, possibly comprising interactions with the surrounding environment or with a user holding the handle. The measurement output of the pressure sensors is regarded as a low-cost indirect torque measurement, and is used in this work for identification and feedback purposes. On the other hand, the more sophisticated load cell is the ground truth measurement providing the exact delivered output torque.

The transmission itself is a passive element (similar to the spring in a SEA actuator) conceived to establish a transparent oneto-one connection between the remotized motor (input shaft) and the robot joint (output shaft). Nominally, we have $T_{o}=T_{m}$, where $T_{o}$ is the torque applied at the robot joint and $T_{m}$ is the torque exerted by the motor. The two hydraulic hoses propagate the power, across long and possibly twisted pathways, in the form of pressure difference between channels $A$ and $B$. Fig. 1 (right) details how the pressure-torque transformation takes place at the output end of the transmission (the same transformation occurs at the input side). Two cylinders are coupled in an antagonistic configuration by the timing belt routed around the output toothed pulley. The cylinders apply a force proportional to their internal pressure on their respective belt branches: when the two pressures (denoted by $p_{A}$ and $p_{B}$ in Fig. 1 (right)) coincide, the two cylinders are balanced and no torque is generated on the pulley; when the two pressures are different, the cylinders are no longer balanced, and thus a net torque $T_{p}$ is generated according to the algebraic relation

$$
T_{p}=A_{e} r \Delta p
$$

where constant $A_{e}$ is the effective area of the cylinders, constant $r$ is the pulley radius, and variable $\Delta p=p_{A}-p_{B}$ is the pressure difference between channels $A$ and $B$. Since the elements connecting the cylinders and the pulley (i.e. the membrane, belts and bearings) are a reasonably low source of friction and hysteresis, then the torque acting on the robot arm can be approximated to be the same as the torque associated to the pressure difference, namely $T_{o} \approx T_{p}$. 
With an incompressible fluid in the hydraulic hoses, the internal volume of the transmission lines remain constant, and, as long as the transmitted torque is small and the operating condition is far from the resonance frequency, the approximated kinematic relation $\theta_{m} \approx \theta_{o}$ is reasonable, where $\theta_{m}$ is the motor angle and $\theta_{o}$ is the output arm angle. In practice, some compliance exists in the transmission system, due to the hoses, belts and diaphragms elasticity, and due to the presence of dissolved air. In particular, in a separate set of tests, we measured a $230 \mathrm{Nm} / \mathrm{rad}$ torsional stiffness between the input and the output shafts for our specific test-bench.

\section{B. Test-Bench Details}

The experimental test-bench was designed to provide at least an angular range of $\theta_{o}=140^{\circ}$ and a maximum transmissible torque of $T_{o}=20 \mathrm{Nm}$. These requirements are representative of a robotic elbow that may be implemented in rehabilitation exoskeletons [11], [17], collaborative robots [16], or similar lightweight robotic applications [20]. Each hydraulic cylinder is equipped with a OA-106-145 rolling diaphragm from DiaCom Corp. characterized by a $24 \mathrm{~mm}$ piston diameter, a 27 mm cylinder diameter, a $56.8 \mathrm{~mm}$ stroke, and a 25 bar maximum working pressure. These cylinders can exert up to a maximum linear force of $1.5 \mathrm{kN}$. Each pair of antagonistic cylinders is coupled by two toothed pulleys having $23.9 \mathrm{~mm}$ primitive radius. The hydraulic hoses are characterised by a $6 \mathrm{~mm}$ inner diameter, and a flexible coating of Kevlar fibers. The testbench is controlled by a CX51 Beckhoff Embedded-PC based on a TwinCAT real-time communication protocol, while an AKM52L direct-drive motor from Kollmorgen (with a rated torque of $8.67 \mathrm{Nm}$, and a peak torque of $19.6 \mathrm{Nm}$ ) is used for the actuation. Distilled water is used as working fluid, and two 3100x pressure sensors from Gems Sensors (with a 16 bar maximum pressure measurement and a 0.04 bar accuracy) are used to measure the fluid pressure. The end-effector, placed at a distance of $b=390 \mathrm{~mm}$ from the arm rotation axis, is equipped with a single-point load cell from Tedea Huntleigh (model $1024,3 \mathrm{~kg}$ capacity), in order to measure the actual output force $F_{o}$; the actual output torque is computed as $T_{o}=b F_{o}$. All of the experiments showed in this work are performed with a fixed sampling rate of $1 \mathrm{kHz}$.

\section{Goal: Pressure Feedback for Torque Control}

In this work we propose a linear model for the transmission system and a novel torque setpoint stabilization control law, using a distal pressure measurement feedback signal along the fluid transmission. This is motivated by the high accuracy (in the range of $1 \%$ of the maximum torque) and large bandwidth $(0-80 \mathrm{~Hz})$ of the output torque estimation that can be ensured by this configuration [3], as compared to the less desirable results of [20] exploiting a proximal sensors placement. The long term purpose of our research activity is the design of a simple and versatile control architecture for human-machine interaction. As a first step towards this end, we focus here on stabilizing a force setpoint for the end-effector. The control design requirements can be summarized as follows:

- Ensure stable closed-loop force setpoint regulation;

- Achieve a low steady-state error;

- Provide a sufficiently damped transient response;

- Provide a large closed-loop bandwidth.

Referring again to the scheme in Fig. 1, the feedback controller should compensate for the disturbances acting "before" the pressure sensing point, such as the viscous friction distributed along the fluid channels, the internal friction of the electrical motor and of the input shaft bearings, the hysteresis of the input membranes, and the motor torque ripple. On the other hand, disturbances injected "after" the sensing point cannot be compensated for. For this reason, the floating-bonnet layout of the cylinders used in [3] is an ideal solution due to the minimal friction losses, thereby minimizing these downstream disturbances: a fact that is confirmed in the experimental results of Section V.

As a final remark, we point out that the force control law is designed under a worst-case condition (from the stability viewpoint), represented by clamping the end effector at a fixed position, so as to interact with an infinitely stiff environment [2]. The designed control law is subsequently tested in a scenario where a human operator interacts with the transmission end-effector through a handle. In this way, enhanced actuation features of the closed-loop system can be proved in a real-life application scenario.

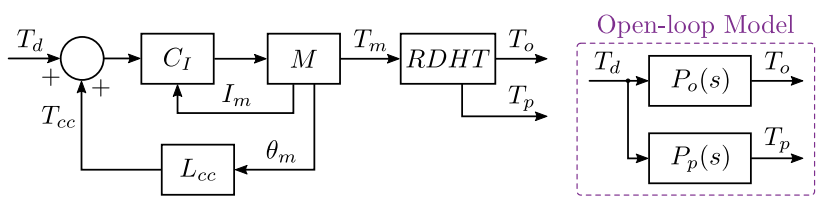

Fig. 2. Scheme of the open-loop plant and its frequency-domain model. 

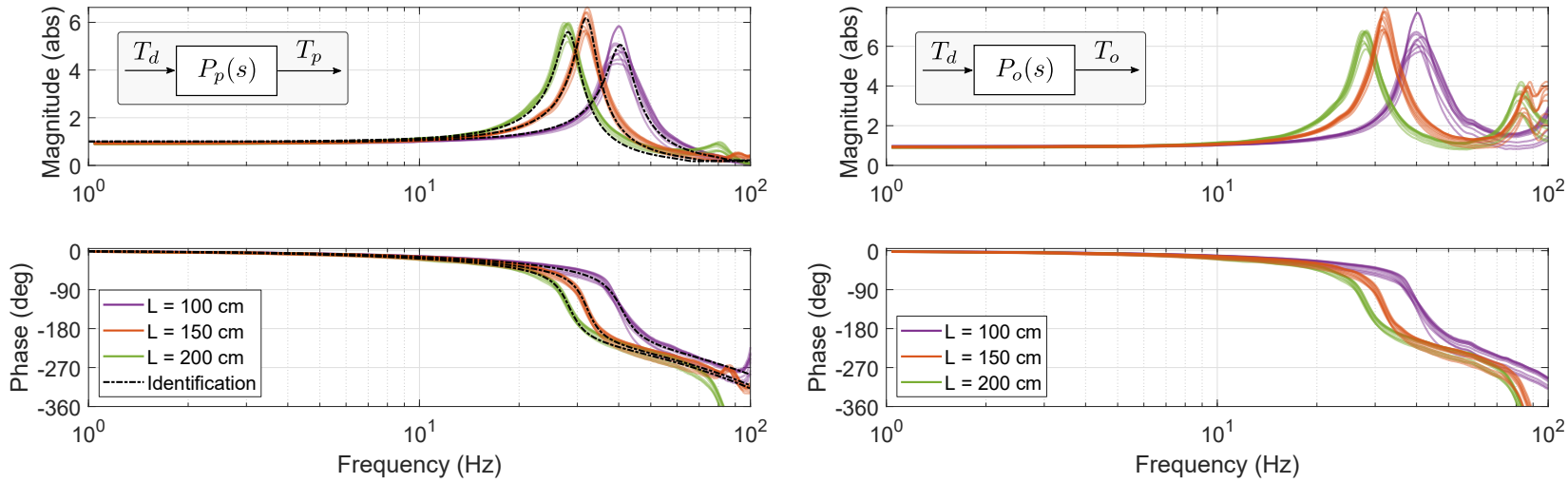

Fig. 3. Experimental frequency responses of the open-loop plant. Left: transfer function $P_{p}(s)$ from $T_{d}$ to $T_{p}$. Right: transfer function $P_{o}(s)$ from $T_{d}$ to $T_{o}$. The experiments are performed using 3 different lengths of the hydraulic hoses $(L=100,150,200 \mathrm{~cm})$. Each color represents a family of linearity experiments, where the test is repeated by setting different values for the input bias $(0,0.5$ and $1 \mathrm{Nm})$ and amplitude $(0.35,0.5$ and $0.75 \mathrm{Nm})$. The dashed-dotted black lines in the left plot represent the identified transfer functions for each one of the considered configurations.

\section{SySTEM MODELING AND IDENTIFICATION}

With reference to Fig. 2, we call open loop the overall dynamical system standing between the desired torque signal $T_{d}$ and the two measured outputs, $T_{p}$ and $T_{o}$, the first one obtained from the pressure sensors, and the second one obtained from the load cell. A transfer function is associated to each output, namely $P_{p}(s)$ from $T_{d}$ to $T_{p}$ (involved in the control design) and $P_{o}(s)$ from $T_{d}$ to $T_{o}$ (used as ground truth validation). Starting from the preliminary results in [3], Fig. 2 shows that the open loop not only pertains to the hardware itself (the motor " $M$ " and the transmission " $R D H T$ "), but also includes the current controller $C_{I}$ executed by the motor driver and a feedback compensation term $T_{c c}$ used to reduce the effect of the cogging torque produced by the electric motor. The cogging-torque compensation waveform $T_{c c}$ is stored as a look-up table, " $L_{c c}$ ", and evaluated based on the measured motor angle $\theta_{m}$.

This section presents the experimental characterisation of the open-loop transfer functions $P_{p}(s)$ and $P_{o}(s)$, and the identification of $P_{p}(s)$, used as a starting point to design a model-based torque controller in Sect. IV. The experiments are repeated for three different lengths $L$ of the hydraulic hoses, since the influence of the hose lengths is a key parameter when the proposed architecture is employed to relocate the actuators of different joints in a robotic system, from those close to the robot base to those placed at distant links. A linearity analysis is also presented.

\section{A. Open-loop Experimental Characterisation}

The identification experiments are performed by clamping the output link to the ground by means of the load cell located at the end effector (the output handle shown in Fig. 1 is removed in this case). For this test, we select the input signal $T_{d}$ as a linear chirp with frequency ranging from 0 to $100 \mathrm{~Hz}$, while the responses of two outputs, $T_{p}$ and $T_{o}$, are simultaneously acquired. Fig. 3 shows the experimental open-loop transfer functions, $P_{p}(s)$ and $P_{o}(s)$, collected for three different lengths of the hydraulic hoses, namely $L=100 \mathrm{~cm}$ (in purple), $L=150 \mathrm{~cm}$ (in red), and $L=200 \mathrm{~cm}$ (in green). The colored curves actually represent a family of experiments, where the identification test has been repeated by setting different values for the input bias $(0,0.5$ and $1 \mathrm{Nm})$ and amplitude $(0.35,0.5$ and $0.75 \mathrm{Nm})$.

The resulting curves confirm the linearity of the dynamics for both measurement outputs. We first note that the magnitude of both transfer functions is well placed around 1 in the range $0-10 \mathrm{~Hz}$, thereby showing good correspondence between the desired torque $T_{d}$ (the input) and the delivered torque $T_{p}$ or $T_{o}$ (the outputs). Above $10 \mathrm{~Hz}$, the dynamic transparency of the transmission is degraded by the first resonance peak, located in the range $25-40 \mathrm{~Hz}$, depending on the hose lengths. As the length $L$ increases, some compliance (mainly due to distributed deformation of the hoses wall) emerges, and the resonance peak takes place at lower frequencies. Since the mechanical dissipative effects are minimized through the adoption of low-friction floating-bonnets, the resonance peak is only lightly damped. A second resonance peak is also visible by the load cell, $P_{o}(s)$, above $80 \mathrm{~Hz}$, which is hardly visible from the pressure sensors measurement. Looking at the phases of the red and green curves, it seems that the location of the pressure sensor plays a role (perhaps from a zero-pole cancellation) for the absence of this peak in $P_{p}(s)$, probably due to further dynamic effects taking place between the pressure sensors and the load cell. Since these high frequency peaks are hardly observable from the pressure sensors, they will affect the high-frequency output performance without being involved in the closed-loop stability considerations.

Finally, it is possible to observe that both phase plots exhibit the typical exponential shape (a linear shape in logarithmic scale) characterizing a pure time-delay phenomenon, which should be taken into account in the considered dynamical model. The source of this time delay, discussed in the next section, is a combination of software processing/sampling effects and possible unmodelled high-frequency dynamics. Moreover, the phase plot of $P_{p}(s)$ and $P_{o}(s)$ have similar values, testifying that the same time delay affects both the pressure and the load-cell outputs. 


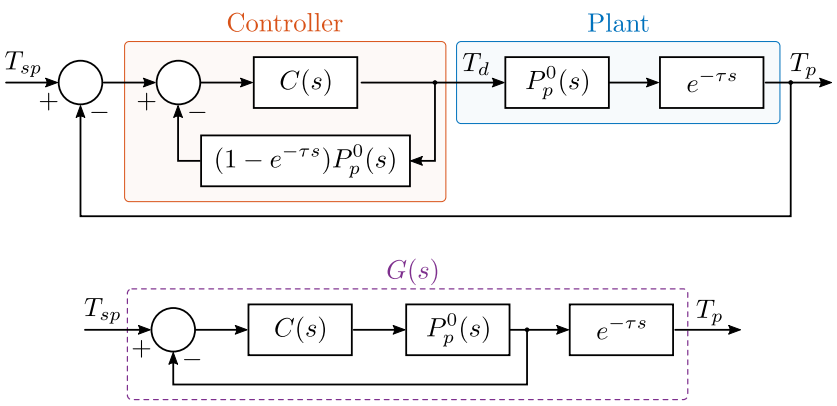

Fig. 4. Proposed control scheme. Top: actual control scheme. Bottom: equivalent closed-loop block diagram due to the Smith predictor.
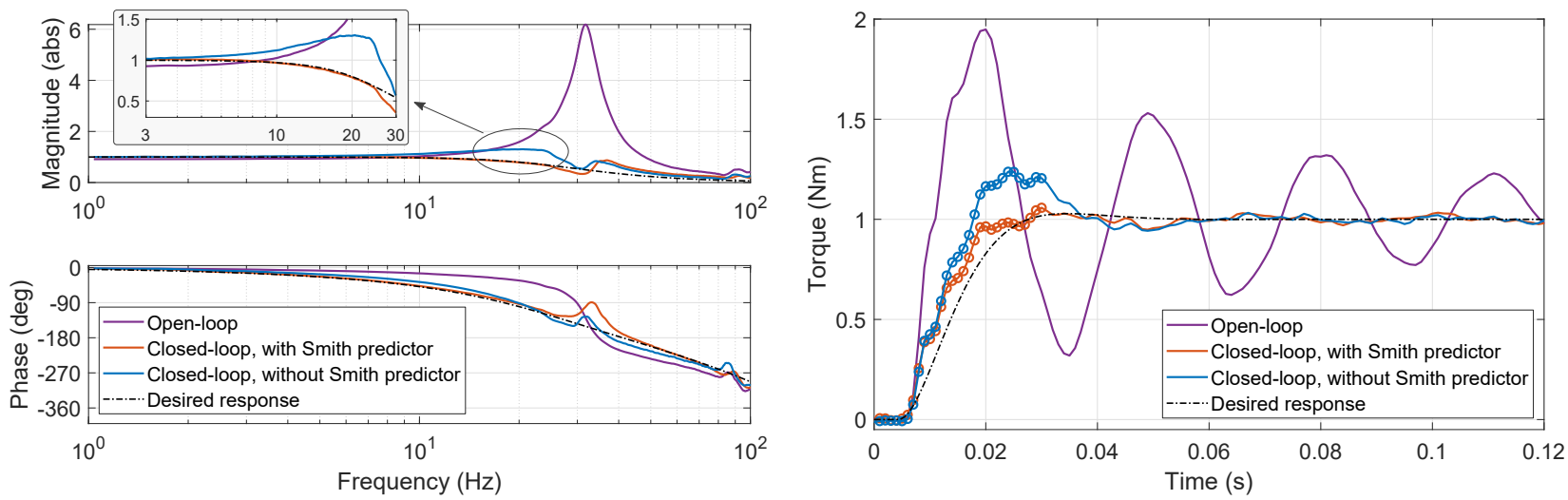

Fig. 5. Effect of the Smith Predictor. The purple line corresponds to the open-loop response; the blue and the red lines correspond to the closed-loop system, but only in the second case the filtered PID controller is augmented with the Smith predictor architecture; the dashed-dotted black line is the target closed-loop response $G_{t}(s)$ in (8). Left: frequency response from setpoint torque $T_{\mathrm{sp}}$ to the measurement $T_{p}$ (from $T_{d}$ to $T_{p}$ in the open-loop case). Right: step response of torque $T_{p}$ measured by the pressure sensors; the circles show the first 30 samples after the step command was executed.

\section{B. Dynamics Parameters Identification}

The analysis carried out in the previous section suggests that a linear second-order model with unit gain and a pure time-delay $\tau$ can appropriately describe the plant $P_{p}(s)$. In particular, we select the reference model

$$
P_{p}(s)=P_{p}^{0}(s) e^{-\tau s}=\frac{\omega^{2}}{\omega^{2}+2 \xi \omega s+s^{2}} e^{-\tau s},
$$

where $P_{p}^{0}(s)$ is a delay-free second order dynamics and $e^{-\tau s}$ provides the pure time-delay component. The parameters in (2) can be identified via standard practices, by minimizing the standard deviation of the difference between the experimental data and the analytical model in the frequency domain. The dashed black lines in Fig. 3 show the response of the identified model (2) for each one of the three considered hose lengths. The identified parameters are reported in Table I. These parameters,

TABLE I

DYNAMicAl PARAMETERS IDENTIFICATION.

\begin{tabular}{cccc}
\hline Configuration & $\omega(\mathrm{Hz})$ & $\xi(-)$ & $\tau(\mathrm{ms})$ \\
\hline$L=100 \mathrm{~cm}$ & 40.86 & 0.0994 & 3.10 \\
$L=150 \mathrm{~cm}$ & 32.02 & 0.0831 & 3.75 \\
$L=200 \mathrm{~cm}$ & 28.35 & 0.0898 & 3.94 \\
\hline
\end{tabular}

which well match the experimental data in all of the analyzed configurations, appear to be acceptable given the low damping of the system and the value of time delay, that increases with the length of the hoses. Table I suggests that the time delay is composed of a constant software contribution and a physical component stemming from the unmodelled and/or high-frequency dynamic effects.

To avoid overloading the presentation, the next sections focus on the configuration corresponding to $L=150 \mathrm{~cm}$ because the other ones, with the corresponding identified parameters from Table I, lead to analogous results. 

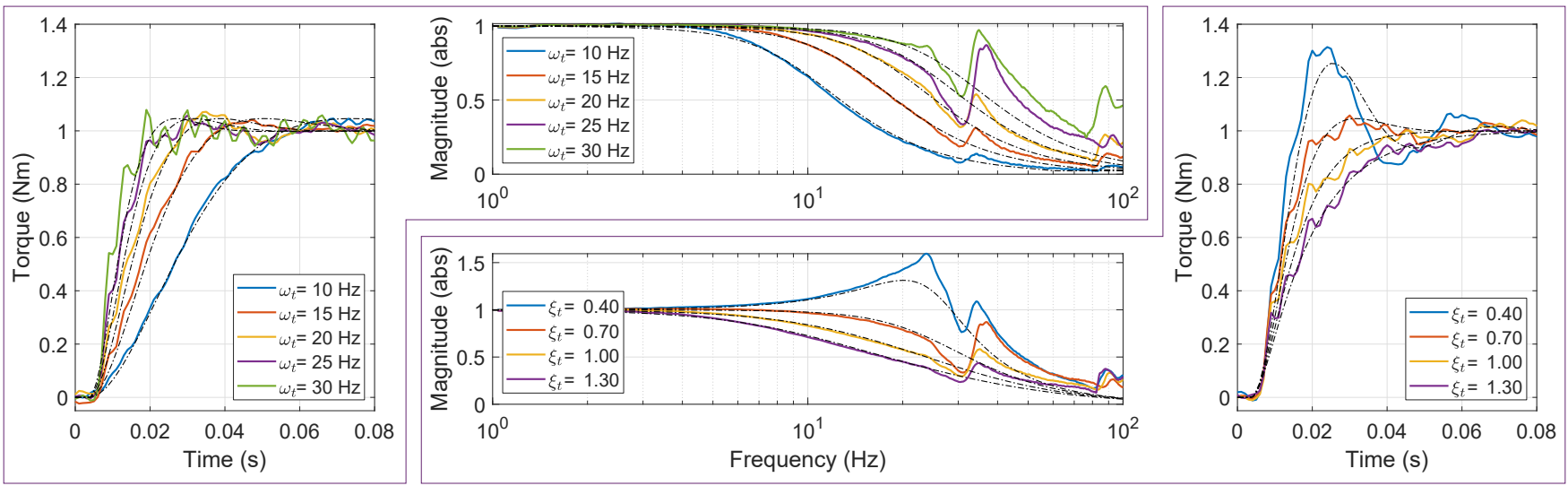

Fig. 6. Closed-loop frequency responses and step responses; the dashed-dotted black lines represent the target responses corresponding to $G_{t}(s)$ in (8). Top and Left: responses with different choices of $\omega_{t}$ and $\xi_{t}=\sqrt{2} / 2$. Bottom and Right: responses with different choices of $\xi_{t}$ and $\omega_{t}=25 \mathrm{~Hz}$.

\section{TORQUE CONTROL}

\section{A. Controller design}

To achieve the design goals summarized in Section II-C, we adopt the Smith-predictor-based control scheme represented in Fig. 4. The open-loop plant (or simply "Plant" as indicated in the figure), is the transfer function $P_{p}(s)$ modeled in Section III as a cascade connection of $P_{p}^{0}(s)$ and $e^{-\tau s}$, according to (2). In order to deal with the time delay, we first select a feedback controller $C(s)$ designed by only focusing on the delay-free transfer function $P_{p}^{0}(s)$, and then augment the scheme with a classical Smith predictor [14], corresponding to the incremental transfer function $\left(1-e^{-\tau s}\right) P_{p}^{0}(s)$. The overall scheme is well represented in Fig. 4 (top) and corresponds to the control law

$$
T_{d}(s)=\frac{C(s)}{1+C(s)\left(1-e^{-\tau s}\right) P_{p}^{0}(s)}\left(T_{\mathrm{sp}}(s)-T_{p}(s)\right),
$$

where $T_{\mathrm{sp}}(s)$ denotes the torque setpoint, and the input to the controller is the setpoint regulation error $T_{\mathrm{sp}}(s)-T_{p}(s)$. Due to the special structure of the Smith predictor, the block diagram in Fig. 4 (top) can be proved to be equivalent to the one in Fig. 4 (bottom). As a result of the Smith predictor, the time delay $\tau$ is removed from the feedback loop and does not play any role in the stability analysis, thereby greatly simplifying the stability and performance-oriented control design. The equivalent closed-loop transfer function from the closed-loop setpoint reference $T_{\mathrm{sp}}(s)$ to the measurement output $T_{p}(s)$, denoted as $G(s)$, can be computed as follows:

$$
G(s)=\frac{T_{d}(s)}{T_{\mathrm{sp}}(s)}=\frac{P_{p}^{0}(s) C(s)}{1+P_{p}^{0}(s) C(s)} e^{-\tau s} .
$$

The closed-loop transfer function (4), clearly indicates that $C(s)$ can be designed depending on $P_{p}^{0}(s)$ only, without needing to account for the time delay.

The particular structure of $P_{p}^{0}(s)$ in (2) suggests a possible candidate $C(s)$ in the form of a filtered PID, as follows:

$$
C(s)=k_{p}+\frac{k_{i}}{s}+\frac{k_{d} s}{T_{f} s+1} .
$$

The low-pass filter time constant $T_{f}$ is introduced to reduce the noise amplification effects of the derivative action, and is considered as a free design parameter alongside gains $k_{p}, k_{i}$, and $k_{d}$. For mathematical convenience, (5) is rewritten in the following alternative form:

$$
C(s)=\frac{\bar{k}_{d} s^{2}+\bar{k}_{p} s+\bar{k}_{i}}{s\left(T_{f} s+1\right)}=\bar{k}_{d} \frac{s^{2}+\frac{\bar{k}_{p}}{k_{d}} s+\frac{\bar{k}_{i}}{k_{d}}}{s\left(T_{f} s+1\right)},
$$

with $\bar{k}_{p}=k_{p}+k_{i} T_{f}, \bar{k}_{i}=k_{i}$, and $\bar{k}_{d}=k_{d}+k_{p} T_{f}$. These three gains can be conveniently selected to ensure a cancellation between the denominator of $P_{p}^{0}(s)$ in (2) and the numerator of the right expression in (6). Then, by choosing all the coefficients as

$$
\bar{k}_{p}=\frac{\xi \omega_{t}}{\xi_{t} \omega}, \bar{k}_{i}=\frac{\omega_{t}}{2 \xi_{t}}, \bar{k}_{d}=\frac{\omega_{t}}{2 \xi_{t} \omega^{2}}, T_{f}=\frac{1}{2 \xi_{t} \omega_{t}},
$$

where $\xi_{t}$ and $\omega_{t}$ are free design parameters (the "target" parameters), the fourth order closed-loop transfer function in (4) exhibits stable zero-pole cancellations and corresponds to

$$
G(s)=G_{t}(s)=\frac{\omega_{t}^{2}}{s^{2}+2 \xi_{t} \omega_{t} s+\omega_{t}^{2}} e^{-\tau s},
$$



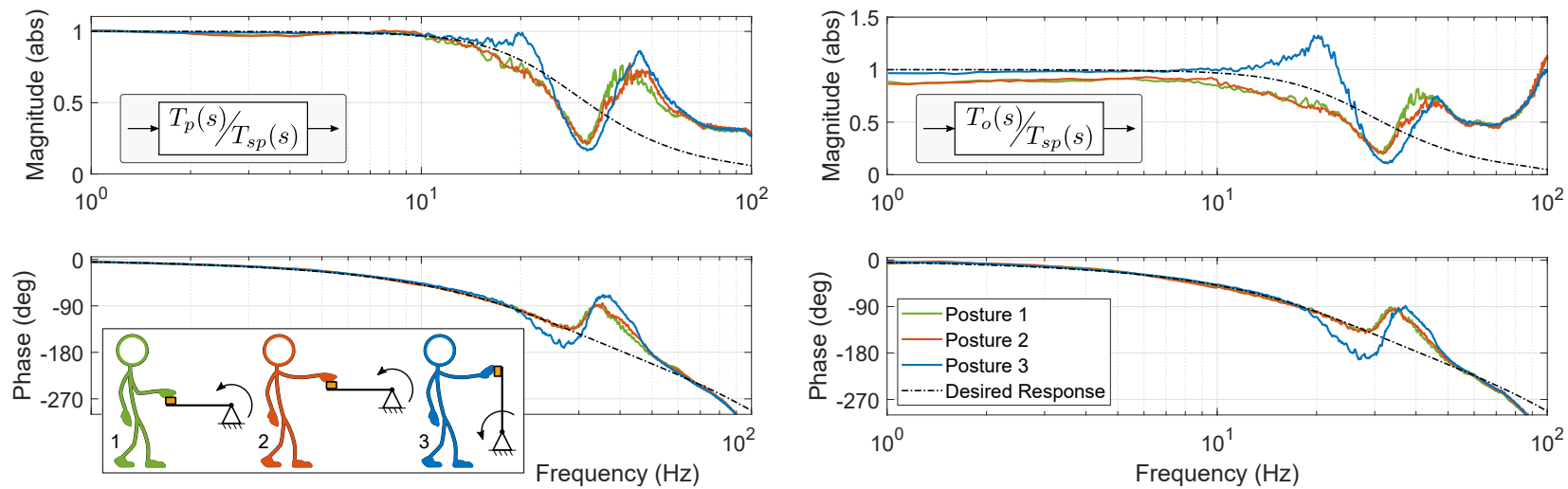

Fig. 7. Closed-loop frequency response in case of interaction with a human user. Different postures of the user body are considered: In posture 1 (green) the user elbow is bent and the forearm is parallel to the robot link; in posture 2 (red) the user arm is straight and parallel to the robot link; in posture 3 (blue) the user arm is straight and perpendicular to the robot link.

which is appealing from the tuning viewpoint. Indeed, with this controller selection, the closed-loop system behaves like a delayed second order transfer function with unitary static gain. The resulting natural frequency $\omega_{t}$ and damping coefficient $\xi_{t}$ can be freely chosen by the designer, while the delay $\tau$ coincides with the open-loop one.

\section{B. Torque Control Experiments}

This section assesses the effectiveness of the proposed control architecture by testing the closed-loop torque-control response $G(s)$, that corresponds to (4), which simplifies to (8) due to the special selection of the PID controller parameters (7) leading to a stable zero-pole cancellation. The controller tuning examined in this section corresponds to the design parameters selection $\xi_{t}=\sqrt{2} / 2 \approx 0.7$, critically damped, and $\omega_{t}=25 \mathrm{~Hz}$ (resulting in controller parameters: $\bar{k}_{p}=0.0918, \bar{k}_{i}=111.07$, $\left.\bar{k}_{d}=0.0027, T_{f}=0.0045\right)$. The critical damping value provides a theoretical overshoot of $4 \%$, a phase margin of 65 degrees, and no resonance, thus it is particularly attractive for the achievement of the specifications reported in Sec. II-C.

In this set of experiments the output link is again fixed to the ground by means of the load cell (same experimental configuration used for the identification of Sect. III-A). Fig. 5 shows the closed-loop frequency response (left) and step response (right). The target response, $G_{t}(s)$ in (8), is represented by dashed-dotted black lines in both plots. The experimental responses are generated both with (solid red line) and without (solid blue line) the Smith predictor (the latter architecture considers only $C(s)$ as the feedback controller, according to Fig. 4 (top)). By comparing the plots, the effectiveness of our PID tuning and the benefits provided by the Smith predictor architecture can be readily observed; the resonance peak is effectively attenuated, and a close match between the target and the experimental frequency responses is achieved up to $25 \mathrm{~Hz}$, Fig. 5 (left). The frequency response also shows that some mismatch occurs in the zero-pole cancellation strategy, which testify that some accuracy limitations of the linear model arise around the resonance, possibly due to some nonlinear and unmodeled dynamics. Additionally, the step responses in Fig. 5 (right) illustrate the improvement obtained by the feedback action from the pressure sensors, as compared to the oscillatory open-loop response. Note also that, as predicted by (8), the open-loop and closed-loop responses have the same time delay with respect to the initial instant $t=0$ of the step.

Finally, Fig. 6 shows that the proposed control architecture allows shaping a large variety of target closed-loop transfer functions $G_{t}(s)$ in (8). In particular, parameter $\omega_{t}$, related to the closed-loop bandwidth, can be increased from low values up to values approaching the natural frequency of the plant $P_{p}(s)$ (despite the significant artificial damping injected to remove the undesired open-loop oscillations). Even if the closed loop remains stable for $\omega_{t}=30 \mathrm{~Hz}$, some vibrations occur, so we can reasonably regard $\omega_{t}=25 \mathrm{~Hz}$ as the maximum achievable closed-loop natural frequency. Fig. 6 (bottom and right) shows that the damping ratio $\xi_{t}$ can also be easily adjusted to reduce the lightly damped open-loop response, ranging from under-damped to over-damped. Each selection produces an experimental response close to the target one given by (8) (dashed-dotted black lines).

\section{HUMAN-ROBOT-INTERACTION}

As a first step towards control of interacting hydrostatic transmissions, we evaluate in this section the proposed force controller $\left(\xi_{t}=\sqrt{2} / 2, \omega_{t}=25 \mathrm{~Hz}\right)$ in an experimental human-robot-interaction setting. In this case, the output link is free to move, while the user interacts with the system via the handle located at the end-effector.

The first experiment, shown in Fig. 7, investigates how the interaction with the human user affects the frequency response of the controller designed under the condition of nominal interaction with a rigid environment. A linear chirp setpoint $T_{\mathrm{sp}}$ with frequency sweeping from 0 to $100 \mathrm{~Hz}$ and an amplitude of $0.5 \mathrm{Nm}$ was used as the set-point for the control system, while the user held the output handle in a fixed position. Several postures of the human body were considered in order to range over a 

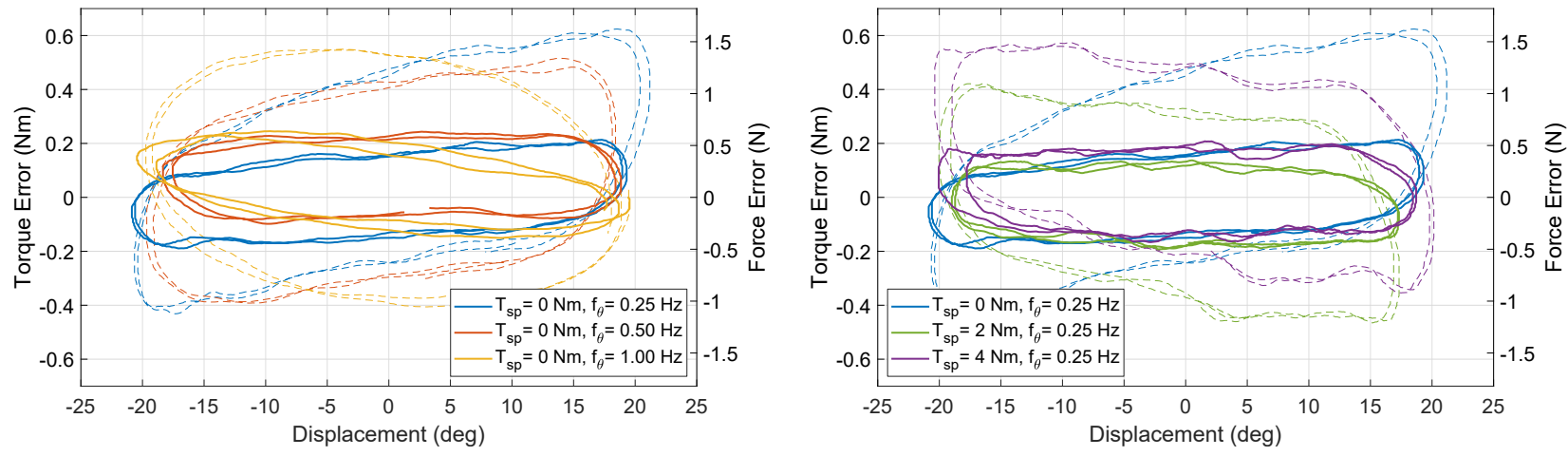

Fig. 8. Backdrivability test. Torque error $T_{o}-T_{\mathrm{sp}}$ measured by the load cell with a sinusoidal displacement of the output link with amplitude $20^{\circ}$, and different displacement frequency $f_{\theta}$ and torque setpoint $T_{s p}$ values. Closed-loop (solid lines) and open-loop (dashed lines) tests are compared.
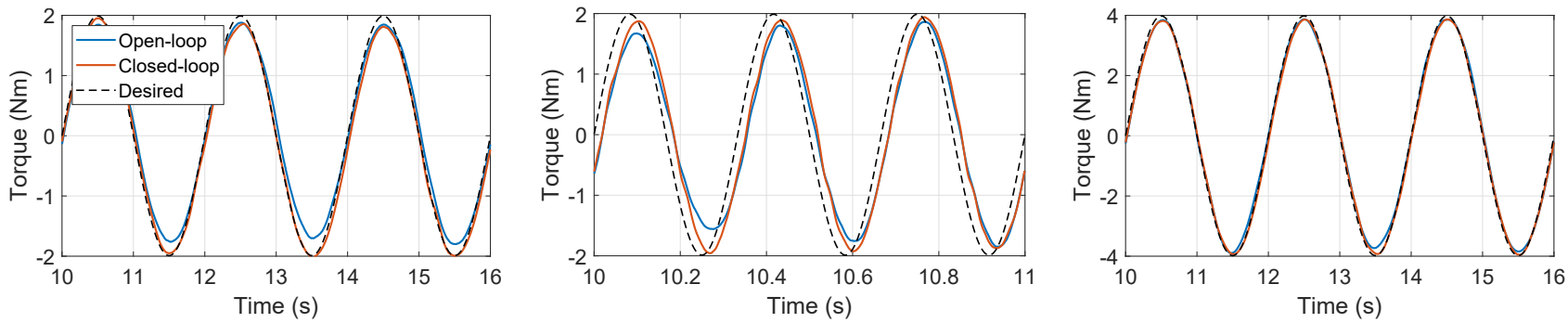

Fig. 9. Measurement of output $T_{o}$ in sinusoidal torque-setpoint $T_{\mathrm{sp}}=A_{\mathrm{sp}} \sin \left(f_{\mathrm{sp}} t\right)$ tracking tests. The robot link is held in a fixed position $\theta_{o}=0^{\circ}$ by the user. The response of open-loop (blue line) and closed-loop (red line) tests are compared. Left: setpoint amplitude $A_{\mathrm{sp}}=2 \mathrm{Nm}$, setpoint frequency $f_{\mathrm{sp}}=0.5 \mathrm{~Hz}$. Center: increased setpoint frequency, $f_{\mathrm{sp}}=3 \mathrm{~Hz}$. Right: increased setpoint amplitude, $A_{\mathrm{sp}}=4 \mathrm{Nm}$.

variety of impedance values that the human body may produce. As a result, the tracking of the pressure reference is almost unaffected by the presence of the human body (left plot), and the overall plant remains stable even in the presence of the output impedance variation. The tests involving postures 1 and 2 reveal that, despite the accurate pressure tracking, the torque measured at the interaction point has a lower amplitude than expected, i.e. $\left|P_{o}(s)\right|<1$ over the entire low-frequency domain. This effect can be justified by noticing that the displacement at the output is no longer negligible due to the compliance of the human arm, and therefore larger friction sources located between the pressure sensing point and the robot link induce $\left|T_{o}\right|<\left|T_{p}\right|$, as discussed at the end of Section II-C. In posture 3 the operator arm offers a stiffer constraint to the robot arm motion, thus, a smaller displacement takes place and, consequently, a better matching between $T_{p}$ and $T_{o}$ is achieved, i.e. $\left|P_{o}(s)\right| \approx 1$. This posture, however, slightly interferes with the resonance attenuation as confirmed by repeated experiments.

The closed-loop low-frequency behavior is further investigated in the next set of experiments. Backdrivability tests were performed by imposing constant torque setpoints, while the user freely moved the output handle on an oscillatory trajectory of amplitude $\approx 20^{\circ}$. Fig. 8 shows the evolution of the output torque error $T_{o}-T_{\mathrm{sp}}$ (as measured by the ground truth load cell) as a function of the displacement angle in two scenarios. At the left, the setpoint $T_{\mathrm{sp}}=0 \mathrm{Nm}$ is associated with different displacement frequencies, specifically $f_{\theta}=0.25,0.5$ and $1 \mathrm{~Hz}$. At the right, different torque setpoints $T_{\mathrm{sp}}=0,2$ and $4 \mathrm{Nm}$ are associated with the frequency $f_{\theta}=0.25 \mathrm{~Hz}$ (so that the blue curves coincide). The two figures report both the openloop (dashed lines) and the closed-loop (solid lines) responses. In all of the considered cases, the control system reduces the maximum value of the torque needed to backdrive the system (namely, the torque error) in the range of $\pm 0.2 \mathrm{Nm}$, thus reducing it by a factor of $\approx 3$ as compared to the open loop. The $y$-axis scale on the right of both figures reports the corresponding force error at the user hand.

Finally, Fig. 9 assesses the tracking of a sinusoidal torque setpoint $T_{s p}=A_{\mathrm{sp}} \sin \left(f_{\mathrm{sp}} t\right)$, while the user holds the robot arm in a fixed position. Several values of the amplitude $A_{\mathrm{sp}}$ and frequencies $f_{\mathrm{sp}}$ are tested. At very low frequency (left plot) the controller action effectively compensates for the friction distributed along the fluid lines as well as for the disturbances located at the motor side, so that the desired torque amplitude is restored at the output. The tracking error does not appear to increase with higher setpoint amplitudes (right plot), thus preserving the quality of the torque rendering under a large range of load conditions. As expected by the Smith predictor architecture, at higher frequencies (central plot) the effect of the time delay $\tau$ is still visible, but the desired amplitude is significantly restored.

\section{CONClusions}

This work investigated the maximum performance limits that one can reasonably expect from RDHTs. We addressed setpoint torque regulation on an RDHT actuation system, via suitable modeling and feedback control techniques, using a cost-effective 
solution exploiting an indirect pressure difference measurement. The experimental results confirm the effectiveness of the proposed torque control system both in grounded end-effector configuration and in human-robot-interaction tests; the oscillatory response of the transmission is effectively attenuated, the settling time of the feedback output is reduced by $95 \%$, and the closed-loop torque bandwidth reaches almost the same natural frequency as the open-loop plant, yet without exhibiting any overshoot at the resonance. The low-friction feature of our floating-bonnet layout reduces the backdrivability torque by almost one order of magnitude as compared to existing RDHT robotic architectures of similar size [19]. Future work comprises providing improved setpoint regulation via adaptive versions of the Smith-predictor-based feedback, in addition to studying the stability of the human-robot-interaction paradigm.

\section{REFERENCES}

[1] Alin Albu-Schäffer, Christian Ott, and Gerd Hirzinger. A unified passivity-based control framework for position, torque and impedance control of flexible joint robots. The international journal of robotics research, 26(1):23-39, 2007.

[2] Chae H An and John M Hollerbach. Dynamic stability issues in force control of manipulators. In IEEE ACC, pages 821-827, 1987.

[3] Marco Bolignari and Marco Fontana. Design and experimental characterization of a high performance hydrostatic transmission for robot actuation. Meccanica, pages 1-11, 2020.

[4] Natalie Burkhard, Samuel Frishman, Alexander Gruebele, J Peter Whitney, Roger Goldman, Bruce Daniel, and Mark Cutkosky. A rolling-diaphragm hydrostatic transmission for remote mr-guided needle insertion. In IEEE int. conf. on robot. and autom., pages 1148-1153, 2017.

[5] Andrea Calanca, Riccardo Muradore, and Paolo Fiorini. A review of algorithms for compliant control of stiff and fixed-compliance robots. IEEE/ASME Transactions on Mechatronics, 21(2):613-624, 2015.

[6] Dongyang Chen, Youngmok Yun, and Ashish D Deshpande. Experimental characterization of bowden cable friction. In 2014 IEEE international conference on robotics and automation (ICRA), pages 5927-5933, 2014.

[7] Stefano Chiaverini, Bruno Siciliano, and Luigi Villani. A survey of robot interaction control schemes with experimental comparison. IEEE/ASME Transactions on mechatronics, 4(3):273-285, 1999.

[8] Marco Fontana, Salsedo Fabio, Simone Marcheschi, and Massimo Bergamasco. Haptic hand exoskeleton for precision grasp simulation. Journal of Mechanisms and Robotics, 5(4), 2013.

[9] F. Freudenstein. On the conceptual design of a novel class of robot configurations. Journal of Mechanisms, Transmissions, and Automation in Design, 111(1):47-53, 1989.

[10] Gerd Hirzinger, A Albu-Schaffer, M Hahnle, Ingo Schaefer, and Norbert Sporer. On a new generation of torque controlled light-weight robots. In IEEE Int. Conf. on Robotics and Automation, pages 3356-3363, 2001.

[11] Bongsu Kim and Ashish D Deshpande. An upper-body rehabilitation exoskeleton harmony with an anatomical shoulder mechanism: Design, modeling, control, and performance evaluation. The International Journal of Robotics Research, 36(4):414-435, 2017.

[12] Thomas H Massie, J Kenneth Salisbury, et al. The phantom haptic interface: A device for probing virtual objects. In Proceedings of the ASME symposium on haptic interfaces for virtual env. and teleop. systems, volume 55, pages 295-300. Chicago, IL, 1994.

[13] Nicholas Paine, Sehoon Oh, and Luis Sentis. Design and control considerations for high-performance series elastic actuators. IEEE/ASME Transactions on Mechatronics, 19(3):1080-1091, 2013.

[14] Z.J. Palmor. Time-delay compensation smith predictor and its modifications. In W.S. Levine, editor, The Control Handbook, pages 224-237. CRC press, USA, 1996.

[15] Eric Schwarm, Kevin M Gravesmill, and John P Whitney. A floating-piston hydrostatic linear actuator and remote-direct-drive 2-dof gripper. In IEEE int. conf. on robot. and autom., pages 7562-7568, 2019.

[16] Dongjun Shin, Irene Sardellitti, Yong-Lae Park, Oussama Khatib, and Mark Cutkosky. Design and control of a bio-inspired human-friendly robot. The International Journal of Robotics Research, 29(5):571-584, 2010.

[17] Thomas G Sugar, Jiping He, Edward J Koeneman, James B Koeneman, Richard Herman, H Huang, Robert S Schultz, DE Herring, J Wanberg, Sivakumar Balasubramanian, et al. Design and control of rupert: a device for robotic upper extremity repetitive therapy. IEEE transactions on neural systems and rehabilitation engineering, 15(3):336-346, 2007.

[18] Catherine Véronneau, Jean-Philippe Lucking Bigué, Alexis Lussier-Desbiens, and Jean-Sébastien Plante. A high-bandwidth back-drivable hydrostatic power distribution system for exoskeletons based on magnetorheological clutches. IEEE Robotics and Automation Letters, 3(3):2592-2599, 2018.

[19] Catherine Véronneau, Jeff Denis, Louis-Philippe Lebel, Marc Denninger, Vincent Blanchard, Alexandre Girard, and Jean-Sébastien Plante. Multifunctional remotely actuated 3-dof supernumerary robotic arm based on magnetorheological clutches and hydrostatic transmission lines. IEEE Robotics and Automation Letters, 5(2):2546-2553, 2020.

[20] Catherine Véronneau, Jeff Denis, Louis-Philippe Lebel, Marc Denninger, Jean-Sébastien Plante, and Alexandre Girard. A lightweight force-controllable wearable arm based on magnetorheological-hydrostatic actuators. In 2019 int. conf. on robotics and automation (ICRA), pages 4018-4024, 2019.

[21] John P Whitney, Tianyao Chen, John Mars, and Jessica K Hodgins. A hybrid hydrostatic transmission and human-safe haptic telepresence robot. In IEEE int. conf. on robotics and autom. (ICRA), pages 690-695, 2016.

[22] John P Whitney, Matthew F Glisson, Eric L Brockmeyer, and Jessica K Hodgins. A low-friction passive fluid transmission and fluid-tendon soft actuator. In IEEE Int. Conf. on Intell. Robots and Sys., pages 2801-2808, 2014. 